\title{
Arthroscopic Lateral Collateral Ligament Repair for the Acute Elbow Dislocation in Primary Lateral Sclerosis Patient: A Case Report
}

\author{
Nattakorn Mahasupachai ${ }^{1}$ (D), Arnakorn Premsiri ${ }^{1}$ (D), Cholawish Chanlalit $^{1}$ (D) ${ }^{\text {a }}$ \\ 1 Orthopedics, Srinakharinwirot University, Nakhon Nayok, Thailand \\ Keywords: primary lateral sclerosis, neuromuscular disease, elbow dislocation, arthroscopic lateral collateral ligament repair \\ https://doi.org/10.52965/001c.32287
}

\section{Orthopedic Reviews}

Vol. 14, Issue 1, 2022

\section{Introduction}

Without adequate muscle function as the dynamic elbow stabilizer, in a patient with a neuromuscular disorder, such as primary lateral sclerosis (PLS), the osteoligamentous structures are the only elbow stabilizers. To provide elbow stability, these structures should be restored.

\section{Case}

A 30-year-old female with PLS suffered from pain and dysfunction of her elbow after a previous elbow injury and current elbow dislocation.

\section{Management and outcomes}

We performed an arthroscopic lateral collateral ligament repair. At 6 months postoperative, her elbow gained stability and a painless full range of motion.

\section{Conclusion}

Periarticular muscles are crucial in joint congruity maintenance when the static stabilizers are disrupted. However, in neuromuscular diseases, the dynamic stabilizers are inadequate in providing stability for ligament healing, causing elbow instability. Osteoligamentous stabilizers restoration is the key to joint stability in a paralytic limb. An arthroscopy is a good option restoring collateral ligaments by reducing damage to surrounding muscles.

\section{INTRODUCTION}

The elbow joint comprises both static and dynamic constraints. The static stabilizers constitute mainly the osteoligamentous structures, whereas muscles crossing the elbow joint assist in forming dynamic stability. ${ }^{1,2}$ Following elbow dislocation, when all the static stabilizers are disrupted, forces across the elbow are crucial in maintaining joint congruity. Their tones help provide secondary tension, especially during forearm pronation. In addition, the contraction of elbow flexors and extensors creates a compressive force pulling ulna back to the humerus, reducing the Drop sign. ${ }^{2-4}$
Primary lateral sclerosis (PLS) is a rare neuromuscular disorder affecting the upper motor neurons. It occurs when nerve cells in the motor regions of the cerebral cortex gradually degenerate, impairing voluntary muscle movement. As a result, progressive spastic muscle weakness and gait disturbance are common clinical presentations. ${ }^{5}$ In patients with PLS proceeding elbow dislocation, it is inevitable that the dynamic stabilizers will be attenuated, and hence, there is inadequate stability for the ligamentous structures to heal correctly. Consequently, recovery of the elbow joint back to its pre-injury status is unfeasible. Therefore, reparation of such osteoligamentous stabilizers is believed to provide restoration of joint congruity and stability. $2,3,5$ We report the immediate successful results of

\footnotetext{
a Corresponding author:

Cholawish Chanlalit

Tel: (+66) 81-837-0705

Email:chanlalit@hotmail.com
} 


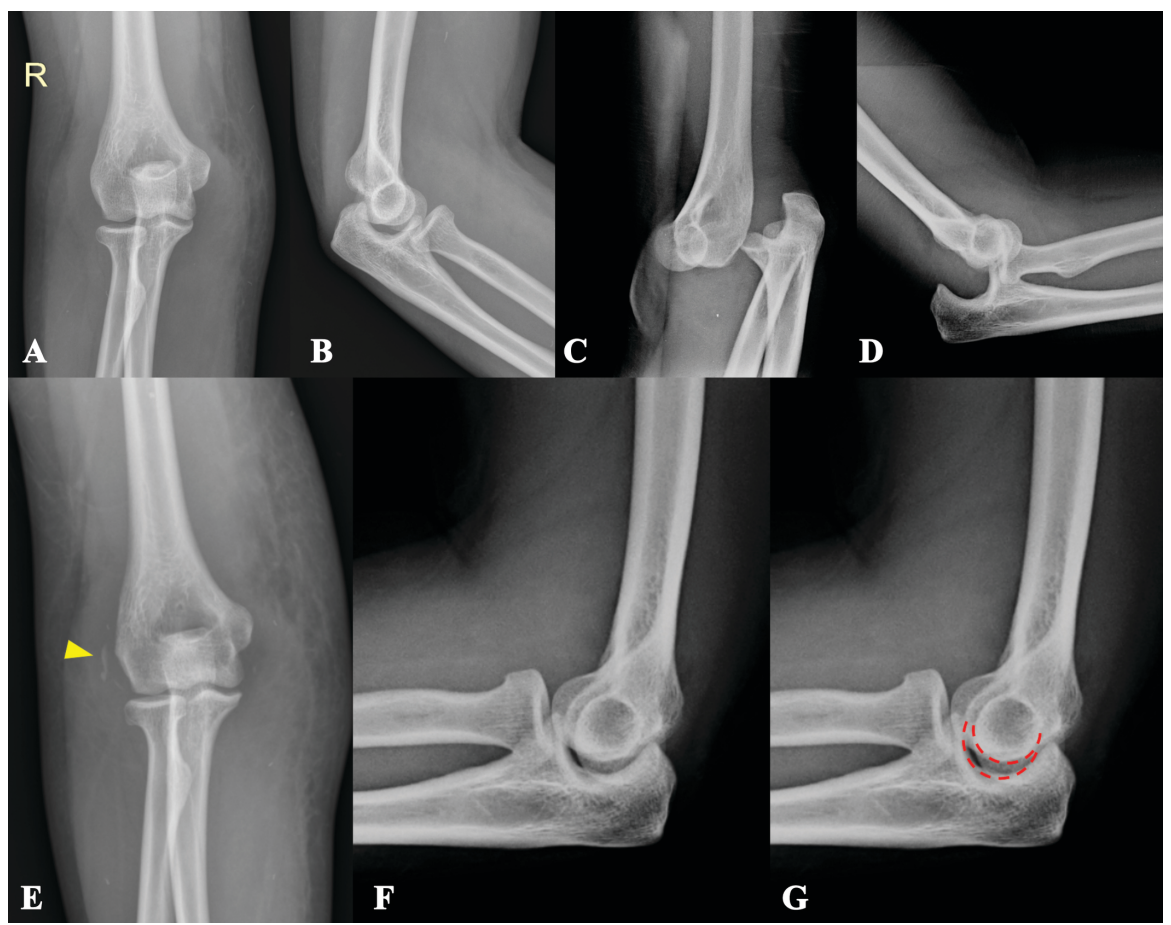

\section{Figure 1.}

A-B) Plain radiographs of patient's right elbow after the previous injury. Drop sign could be seen. C-D) Plain radiographs show right posterior elbow dislocation at the current visit.

E-F) post-reduction radiographs show an avulsion fracture of the lateral epicondyle (arrowhead).

G) The outline shows incongruent ulnohumeral joint and Drop sign.

arthroscopic lateral collateral ligament (LCL) repair in a PLS patient with acute elbow dislocation. The patient provided written consent concerning the publication of her data.

\section{CASE}

A 30-year-old female with a history of 5-year PLS presented with right elbow dislocation 30 minutes before arrival. Her status was generalized muscles weakness requiring wheelchair or gait-aids ambulation. Previously, she had a 3-month history of elbow injury. She recalled an apprehension of right elbow subluxation at that time, which was promptly self-reduced. Visiting her general orthopedist, the slab was applied for two weeks, and after removal, there was no clinical re-dislocation. Even so, she still experienced instability pain on her right elbow up until now. A drop sign was found from reviewing her plain films taken during the previous 3-month follow-up period (Fig. 1A-B).

Due to elbow discomfort and gait-aids-ambulatory status, she suffered another right elbow injury leading to recurrent dislocation at the current visit (Fig. 1C-D). After closed reduction under intra-articular lidocaine injection, a full range of motion with no re-dislocation upon full passive extension was noted. However, a more prominent joint incongruity and an avulsion fracture of the lateral epicondyle were observed (Fig. 1E-G). Subsequently, she was sent to consult with the Sports Upper Extremity unit. Upon evaluation and review of her previous injury history, she still had medial and lateral elbow pain, and acute-on- top-chronic elbow injury was impressed. Accordingly, with an avulsion fracture of the lateral collateral ligament and chronic instability pain, surgical intervention was indicated to restore ligament stability as the compensatory tension from her muscles was suspected to be inferior to that of the general population. 2,3

\section{MANAGEMENT AND OUTCOMES}

In this case, we performed arthroscopic Lateral Collateral Ligament Repair to restore the osteoligamentous structures and provide elbow stability. Perioperatively, examination of the patient's elbow under anesthesia revealed a positive posterolateral drawer test and fluoroscopic varus stress test, further supporting our impression. The patient was then set in a lateral decubitus position with proximal arm support. The sterile drape was prepared from the fingertip up to the tourniquet area, initially applied at her proximal arm. Arthroscopic anterior compartment examination was done via the proximal anteromedial portal, and evidence of capsular tear and joint space widening was recorded (Fig. $\underline{2 \mathrm{~A}})$.

Arthroscopic lavage was performed to remove the cartilaginous loose body. Then we continued with the posterior compartment for lateral collateral ligament repair. The posterior portal was used as a viewing portal, whereas the posterolateral portal worked. As a result, ulnohumeral distance widening and drive-through sign were observed (Fig. 2B). Moreover, a bony avulsion of the LCL from the lateral epicondyle was visualized (Fig. 2C). 


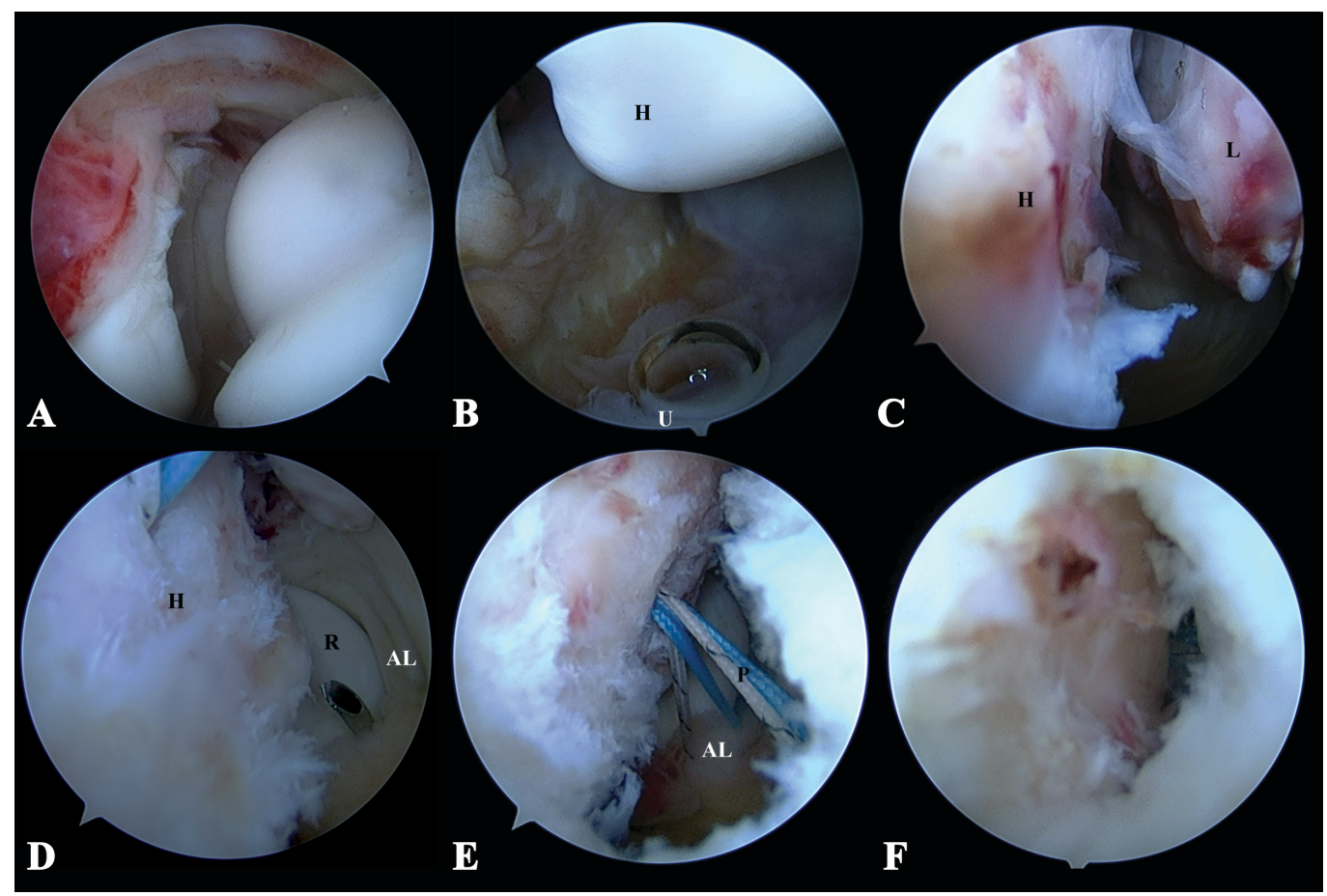

Figure 2.

A) Arthroscopic image reveals capsular hemorrhage and joint space widening.

B) Through the posterior portal, ulnohumeral joint space had widened upon forceful supination.

C) Avulsion of the lateral collateral ligament from the lateral epicondyle of the humerus.

D) Suture passer was inserted through the anconeus fascia and annular ligament.

E) One end from both sutures was passed through the annular ligament and another through the posterolateral portal.

F) The gap between the lateral epicondyle and lateral collateral ligament decreased after knot tying.

( $\mathrm{H}=$ humerus, $\mathrm{L}=$ lateral epicondyle avulsion fragment, $\mathrm{R}=$ radial head, $\mathrm{AL}=$ annular ligament, $\mathrm{P}$ = posterolateral portal)

Next, the LCL humeral footprint and stump were debrided, preparing them for the application of suture anchor. A soft anchor (1.8-mm double-loaded Y-Knot ${ }^{\circledR}$ Flex, ConMed) was inserted at the lateral epicondyle. A small skin incision was made posterior to the radial head and dissected to expose the anconeus fascia. Following annular ligament identification, the suture passer was pierced through the previous incision, first through the anconeus fascia and then the annular ligament (Fig. 2D). One end of the suture anchor was retrieved back to the incision through this tract. The steps mentioned were repeated for another suture strand (Fig. 2E).

A subcutaneous tunnel was created from the previous incision toward the posterolateral portal. The sutures' ends from the posterolateral portal were retrieved from the incision through this tunnel. Lastly, sutures were tied over the anconeus fascia to repair and imbricate the LCL (Fig.2F). After the repair, the drive-through sign and ulnohumeral opening gap were confirmed negative. Likewise, elbow stability was examined, and no subluxation resulted during end extension. The patient was immobilized with a long arm splint, and the elbow joint was congruent after the operation (Fig.3A-B).

Postoperatively, after two weeks, surgical stitches and splint were removed, and an adjustable hinged elbow brace was applied. Passive elbow range of motion exercise was advised. Elbow extension block angle was limited at 30 degrees, whereas flexion angle was not limited. The extension block angle was gradually adjusted to decrease to 0 degrees at four weeks postoperative. The elbow brace was discontinued at six weeks, allowing forearm extensors and flexors to strengthen. Upper limb proprioceptive training, namely the ball-rolling exercise, was then adopted.

At one month postoperative, she reported complete pain recovery with active elbow flexion of 120 degrees, extension of -10 degrees, and negative varus and valgus stress tests. Three months after surgery, her elbow gained full range of motion, 140 degrees flexion, and full extension, without any joint subluxation (Fig. 3C-F). In addition, the posterolateral drawer test and pivot shift apprehension test were negative at this follow-up. At the most recent follow-up, six months postoperative, a visual analog scale of 0, Quick DASH score of 29.5, and Mayo elbow performance index of 95 signify satisfactory functional outcomes.

\section{DISCUSSION}

For individuals living with PLS, elbow stability is dependent exclusively on the osteoligamentous structures. Upon elbow dislocation, these static stabilizers are impaired, and together with the unreliably weakened dynamic secondary constraints, marked instability is very likely. $1-3,5$ The tension of the musculotendinous unit is also different between elbow dislocation stages 3B and 3C, further highlighting the significance of muscles crossing the elbow. Instability events in patients with neuromuscular disorders are rel- 


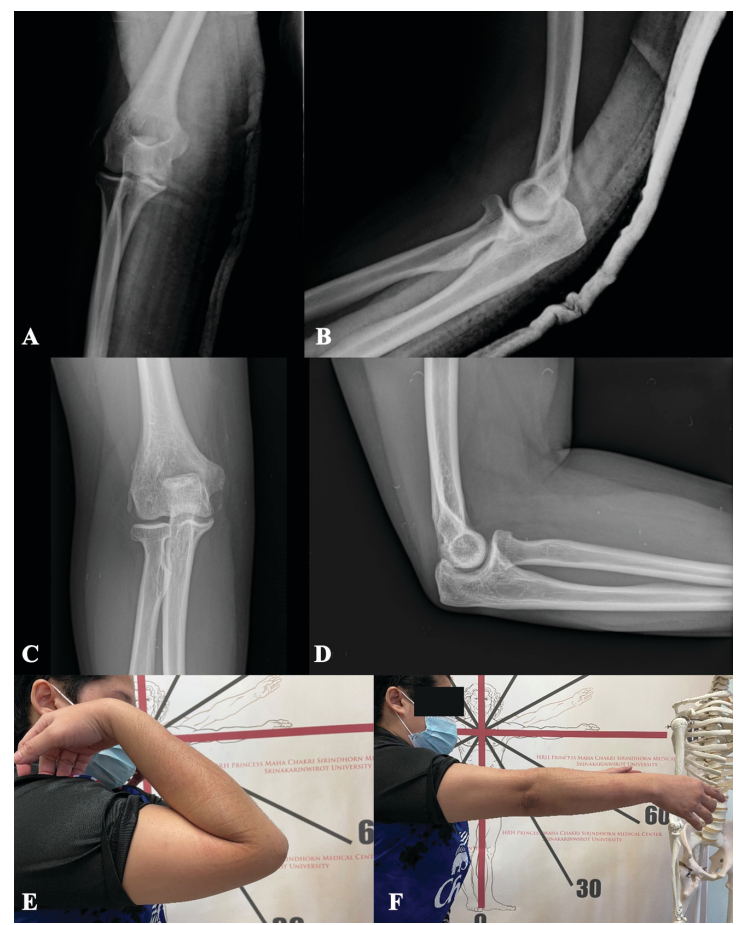

Figure 3.

A-B) Postoperative elbow radiographs show ulnohumeral joint congruency. No Drop sign was presented.

C-D) Plain elbow anteroposterior and lateral radiographs show congruent elbow joint at 6 months postoperative.

E-F) At 3 months postoperative, the patient actively performed 140 degrees elbow flexion and full-extension, similar to the contralateral side.

atively common. For example. ${ }^{2,3,6}$ shoulder subluxation caused by stroke-induced paralysis has an incidence of 17-81\%. Periarticular muscles weakness and the weight of the upper limb are the causes of such occurrence. ${ }^{7}$ Likewise, hip dislocation in Poliomyelitis can arise due to flaccid paralysis and the resulting muscular imbalance. ${ }^{8}$ Suh et al. retrospectively reviewed medical records of 190 patients undergoing bipolar hemiarthroplasty. They found that patients with neuromuscular diseases, specifically cerebral infarction and Parkinsonism, reportedly had a greater postoperative dislocation incidence than those without. Such finding was also observed in cases receiving total hip arthroplasty. ${ }^{9}$ Shim et al. found that the hamstrings and quadriceps act as dynamic agonists for the anterior cruciate ligament (ACL) and play an essential role in preventing anterior subluxation of the knee. Consequently, atrophy of these muscles could exacerbate functional disability. ${ }^{10} \mathrm{Cur}$ rently, there is no literature reporting the association between neuromuscular disorder and the event of elbow subluxation or dislocation. Still, we postulate that the failure of dynamic stabilizers following muscles weakness can also occur equally across the elbow joint.

This case report depicts an improper ligament healing following a 3-month-old injury. Due to her persisting elbow instability from LCL laxity along with the history of repetitive injury, we considered repairing the ligament to restore its integrity as our goal. After arthroscopic LCL repair, her elbow gained stability and a full range of motion without pain, identical to the pre-injury status. The arthroscopy was preferred because it requires less musculature dissection, limiting the attenuation of the already weakened mus- cles in this patient. This idea was supported by Suh et al. nine by maintaining just adequate soft tissue tension using a posterior gentle tissue repairing technique; they found no difference in the incidence of dislocation between patients with and without neuromuscular disease undergoing bipolar hemiarthroplasty. To the best of our knowledge, this study is the first report of a case of an acute elbow dislocation in a PLS patient treated with arthroscopic LCL reconstruction providing excellent results.

\section{CONCLUSION}

In conclusion, repairing or reconstructing the static stabilizers for supreme healing ability is the mainstream treatment concept for a patient with elbow dislocation complicated with PLS or other paralytic muscle disorders. Arthroscopic LCL reconstruction, a minimally invasive procedure, has provided immediate successful results in this patient. Therefore, we consider it one promising treatment option in patients with muscular weakness.

\section{ACKNOWLEDGMENTS}

We appreciated all the officers in the department of orthopaedics for every support and help.

\section{AUTHOR CONTRIBUTIONS}

Category 1 
Conception and design of the study: CC Acquisition of data: NM, AP

Analysis and interpretation of data: NM, AP

Category 2

Drafting the manuscript: NM, AP

Revising the manuscript critically for important intellectual content: CC

Category 3

Approval of the version of the manuscript to be published: NM, CC, AP

\section{INFORMED CONSENT FOR PUBLICATION}

This case report was consented to by the patient.

DECLARATION OF CONFLICTING INTERESTS

All authors declare that there is no conflict of interest. There is no funding from the third party. 


\section{REFERENCES}

1. Karbach LE, Elfar J. Elbow Instability: Anatomy, Biomechanics, Diagnostic Maneuvers, and Testing. The Journal of Hand Surgery. 2017;42(2):118-126. do i:10.1016/j.jhsa.2016.11.025

2. O’Driscoll SW, Jupiter JB, King GJ, et al. The unstable elbow. Instr Course Lect. 2001;50:89-102.

3. O'Driscoll SW, Bell DF, Morrey BF. Posterolateral rotatory instability of the elbow. The Journal of Bone \& Joint Surgery. 1991;73(3):440-446. doi:10.2106/000 04623-199173030-00015

4. Gibbons SGT. Mottram SL. Stability and Movement Dysfunction Related to the Elbow and Forearm. $2001 ; 20$.

5. Turner MR, Barohn RJ, Corcia P, Fink JK, Harms MB, Kiernan MC, et al. Primary lateral sclerosis: Consensus diagnostic criteria. J Neurol Neurosurg Psychiatry. 2020;Apr;91(4):373-7:373-377. doi:10.113 6/jeep-2019-322541

6. Camp CL, Smith J, O’Driscoll SW. Posterolateral Rotatory Instability of the Elbow: Part I. Mechanism of Injury and the Posterolateral Rotatory Drawer Test. Arthroscopy Techniques. 2017;6(2):401-405. doi:10.10 16/j.eats.2016.10.016
7. Fujimura K, Kagaya H, Endou C, et al. Effects of repetitive peripheral magnetic stimulation on shoulder subluxations caused by stroke: A preliminary study. Neuromodulation: Technology at the Neural Interface. 2019;23(6):847-851. doi:10.1111/ne r.13064

8. Laguna R, Barrientos J. Total hip arthroplasty in Paralytic dislocation From Poliomyelitis. Orthopedics. 2008;31(2):179. doi:10.3928/01477447-20080201-16

9. Suh KT, Kim DW, Lee HS, Seong YJ, Lee JS. Is the Dislocation Rate Higher after Bipolar

Hemiarthroplasty in Patients with Neuromuscular Diseases? Clinical Orthopaedics \& Related Research. 2012;470(4):1158-1164. doi:10.1007/s11999-011-213 9-9

10. Shim JK, Choi HS, Shin JH. Effects of neuromuscular training on knee joint stability after anterior cruciate ligament reconstruction. J Phys Ther Sci. 2015;27(12):3613-3617. doi:10.1589/jpts.27.3613 